\title{
Lactococosis en Pseudoplatystoma reticulatum
}

\author{
F. Quintana, C.I.; Guidoli, M.G.; Domitrovic, H.A.; Blanco, T.K.
}

Instituto de Ictiología del Nordeste, Facultad de Ciencias Veterinarias, Universidad

Nacional del Nordeste (UNNE), Cabral 2139, Corrientes (3400), Argentina.

Tel 0379-4425753 interno 152. E-mail: carolina@vet.unne.edu.ar

\begin{abstract}
Resumen
F.Quintana, C.I.; Guidoli, M.G.; Domitrovic, H.A.; Blanco, T.K.: Lactococosis en Pseudoplatystoma reticulatum. Rev. vet. 28: 2, 132-137, 2017. La lactococosis fue reportada a nivel mundial en numerosas especies de peces, ocasionando elevadas pérdidas económicas. Pseudoplatystoma reticulatum es una especie autóctona del río Paraná muy apreciada para el consumo, por lo cual la evaluación de las patologías en los cultivos intensivos es importante. Las muestras obtenidas en este trabajo corresponden a ejemplares juveniles provenientes de una piscicultura de la región nordeste argentina donde se registró una mortandad aguda. Los ejemplares afectados fueron anestesiados y sacrificados. Se realizó la ablación del hígado y secciones del órgano fueron empleadas para improntas. Pequeños trozos se colocaron en medio de cultivo Reinforced Clostridial Medium (Difco) y fueron incubados. El medio de cultivo crecido se sembró por estriado en placas conteniendo medio de Man Rogosa y Sharpe agar (MRS Merck), cultivándose durante 24 a 48 h. Los microorganismos obtenidos se sometieron a pruebas bioquímicas para determinar su ubicación taxonómica. Fragmentos de branquias, hígado, bazo, tubo digestivo y riñón fueron fijados en solución de Bouin, posteriormente fueron deshidratadas e incluidas en parafina. Los cortes histológicos se colorearon con Hematoxilina-Eosina, PAS y Giemsa. Se encontraron lesiones hemorrágicas en distintos puntos del tegumento, presencia de líquido ascítico y numerosas lesiones puntiformes en hígado. En las improntas y pruebas bioquímicas se detectaron cocos Gram positivos aislados, en diplos o en cadenas cortas, catalasas negativas e incapaces de reducir nitratos y sintetizar triptófano a partir de indol. En el hígado se observó predominio de alteraciones vasculares, fundamentalmente hemorrágicas de marcada intensidad. En el bazo se observaron intensas alteraciones vasculares, con predominio congestivo y hemorrágico. Las características clínicas de las lesiones, las pruebas bioquímicas y el diagnóstico histopatológico permiten atribuir la mortandad a Lactococcus garvieae.
\end{abstract}

Palabras clave: pez, bacteria, septicemia hemorrágica.

\begin{abstract}
F.Quintana, C.I.; Guidoli, M.G.; Domitrovic, H.A.; Blanco, T.K.: Lactococcosis in Pseudoplatystoma reticulatum. Rev. vet. 28: 2, 132-137, 2017. Lactococcosis is reported worldwide in numerous fish species causing heavy financial losses. Pseudoplatystoma reticulatum is a native species of Paraná River, edible and highly appreciated in the region, so the consideration of diseases in intensive crops is important. In this work we present a high mortality case of $P$. reticulatum juveniles from a farm located in Northeastern Argentina. For this purpose, animals with symptoms were euthanized and the head was removed. Liver was collected and sections of the organ were used for histopathology. Small pieces were placed in tubes containing Reinforced Clostridial Medium (Difco) and then incubated. Cultures with microorganisms were incubated on plates containing Man Rogosa and Sharpe agar (MRS Merck) during 24 to $28 \mathrm{~h}$. The colonies obtained were restrocked and kept refrigerated at $-20^{\circ} \mathrm{C}$ in media containing glycerol at $20 \%$. Cultured microorganisms were submitted to various biochemical tests to determine their taxonomy. Samples of gills, liver, spleen, gastrointestinal tract and kidney were fixed in Bouin's solution and then dehydrated and embedded in paraffin. Histological sections were stained with Hematoxylin-Eosin, PAS and Giemsa. Hemorrhagic lesions were found in different parts of the tegument, and presence of ascites and numerous punctuate lesions in the liver were observed. In the imprints and biochemical tests of isolates Gram positive cocci were isolated, in diplos or short chains, that were negative catalase test and unable to reduce nitrate and synthesize tryptophan from indol. In the liver it was observed predominance of vascular alterations, mainly hemorrhagic of high
\end{abstract}


intensity. Bacterial invasion stood out with predominantly peripheral location. In the spleen intense vascular alterations were observed with congestive and hemorrhagic dominance. It can be concluded according to the clinical characteristics of lesions, biochemical tests and histopathological diagnosis that mortality of fishes was due to Lactococcus garvieae.

Key words: fish, bacteria, septicaemic infection.

\section{INTRODUCCIÓN}

Lactococcus garvieae es una especie bacteriana de importancia clínica tanto en seres humanos como animales ${ }^{14}$, siendo uno de los agentes causales de mastitis en bovinos y búfalos ${ }^{7,30}$. Fue aislado a partir de muestras clínicas de sangre, orina y piel de pacientes humanos ${ }^{15}$. Tiene amplia distribución en alimentos lácteos y cárneos, por lo cual se lo incluye como un potencial agente zoonótico, existiendo estudios epidemiológicos que evalúan la virulencia de sus diferentes cepas ${ }^{13,16}$, 23,25 .

Entre Lactococcus y Enterococcus existen semejanzas fenotípicas que dificultan su diagnóstico microbiológico. La indisponibilidad de exámenes precisos en la mayoría de los centros médicos del mundo probablemente genere un subdiagnóstico de Lactococcus garvieae el cual podría implicar una significación clínica mucho mayor de la que le es atribuida ${ }^{8,19}$.

En peces, la enfermedad ocasionada por este microorganismo se definió como lactococosis, a fin de diferenciarla de aquellas cuyos agentes etiológicos son otros géneros de cocos Gram positivos y catalasa negativos, como Streptococcus iniae, Streptococcus parauberis o Vagococcus salmoniarum, que se conocen con el término genérico de estreptococosis ${ }^{3,9,10,12,22}$.

Las infecciones por L. garvieae se diagnosticaron en diferentes países ${ }^{11,12,20,21}$ con mayor impacto económico en la industria de la trucha, anguila (Anguilla anguilla), cola amarilla (Chrysiptera parasema) y camarón (Penaeus vannamei) ${ }^{6,10,21}$. Los peces afectados presentan anorexia, melanosis, letargia, pérdida de orientación y natación errática. Los signos externos típicos son exoftalmia, hemorragias en las zonas periorbital e intraocular, base de las aletas y regiones perianal, opercular y bucal.

A pesar del incremento en la significación clínica de L. garvieae, los estudios para su caracterización y relación epidemiológica entre los diferentes aislamientos, sigue siendo muy limitada ${ }^{12,30}$, debiendo aplicarse técnicas bioquímicas rutinarias y/o tests reactivos para poder diferenciar esta patología de otras relacionadas y llegar así a un tratamiento adecuado ${ }^{31}$.

Pseudoplatystoma reticulatum ("surubí atigrado") es un pez autóctono del río Paraná, de interés comercial y deportivo, resultando importante la evaluación de las patologías capaces de afectarlo. En este trabajo se analizan las características clínicas, histopatológicas y microbiológicas que se presentaron en una mortandad aguda registrada en una piscicultura de Pseudoplatystoma reticulatum en la región nordeste argentina.

\section{MATERIAL Y MÉTODOS}

Las muestras obtenidas correspondieron a ejemplares juveniles de Pseudoplatystoma reticulatum de 5 meses de edad, provenientes de un establecimiento comercial ubicado en el nordeste argentino, donde se registró una mortandad aguda. Los ejemplares afectados fueron anestesiados con agua helada y sacrificados por desmedulación. En dos ejemplares se realizó la ablación del hígado en condiciones de esterilidad. Secciones del órgano obtenidas asépticamente se usaron para realizar improntas en portaobjetos limpios y desengrasados.

Los preparados se dejaron secar, se fijaron por acción del calor y se tiñeron mediante coloración de Gram usando el kit de coloración Gram de Britania (código B1446082), aplicando la técnica descripta en el instructivo correspondiente. Una vez secos, los preparados se observaron con objetivo de 100x en un microscopio Olimpus BX41, adaptado a una cámara digital C7070.

Pequeños trozos de los hígados así obtenidos fueron colocados en tubos conteniendo $10 \mathrm{ml}$ de Reinforced Clostridial Medium (Difco) e incubados durante 48 h a $37^{\circ} \mathrm{C}$. El medio de cultivo crecido se sembró por estriado en placas conteniendo agar de Man Rogosa y Sharpe (MRS-Merck) y se cultivó durante 24 a 48 horas a $37^{\circ} \mathrm{C}$. Las colonias obtenidas se repicaron hasta asegurar su pureza y se conservaron refrigeradas a $-20^{\circ} \mathrm{C}$ en medios conteniendo glicerol al $20 \%$ hasta su posterior utilización. Los microorganismos así obtenidos fueron sometidos a diferentes pruebas bioquímicas para determinar su ubicación taxonómica:

Coloración de Gram-Nicolle: se realizó usando el kit de coloración Gram de Britania ya descripto, aplicándose la técnica detallada en el instructivo correspondiente.

Actividad catalasa: en un portaobjetos se mezclaron $20 \mu \mathrm{l}$ de cultivo activo con $20 \mu \mathrm{l}$ de peróxido de hidrógeno 30 volúmenes ${ }^{18}$.

Reducción de $\mathrm{KNO}_{3}$ : las suspensiones de microorganismos se inocularon en el medio específico (peptona de carne $0,86 \%, \mathrm{NaCl} 0,64 \%, \mathrm{KNO}_{3} 0,15 \%, \mathrm{pH} 7,2$.) y se incubaron durante 24 a $48 \mathrm{~h}$ a $37^{\circ} \mathrm{C}$. La producción de nitritos se evidenció por la aparición de color naranja luego de la adición de gotas de solución de $\alpha$-naftol ( $1 \mathrm{~g}$ de $\alpha$-naftol en $100 \mathrm{ml}$ de etanol absoluto) y ácido sulfanílico (1 g de ácido sulfanílico en $100 \mathrm{ml}$ de ácido acético $5 \mathrm{~N}$ ). El nitrato residual se detectó mediante el agregado de polvo de $\mathrm{Zn}$ y la presencia de amonio se evidenció utilizando el reactivo de Nessler ( $7 \mathrm{~g}$ de KI, 
$10 \mathrm{~g}$ de $\mathrm{HgI}_{2}$, y $10 \mathrm{~g}$ de $\mathrm{KOH}$ en $100 \mathrm{ml}$ de agua destilada).

Producción de indol a partir de triptófano: las suspensiones de microorganismos se inocularon en el medio adecuado (peptona de carne $1 \%, \mathrm{D}, \mathrm{L}$-triptófano $0,1 \%, \mathrm{NaCl} 0,5 \%, \mathrm{pH} \mathrm{7,2)} \mathrm{y} \mathrm{se}$ incubaron por 24 a $48 \mathrm{~h}$ a $37^{\circ} \mathrm{C}$. La producción de indol se reveló con el agregado de los reactivos Gracian I (formol y $\mathrm{H}_{2} \mathrm{SO}_{4}$ concentrado en proporción 2:1) y Gracian II $\left(\mathrm{K}_{2} \mathrm{Cr}_{2} \mathrm{O}_{3}\right.$ al $\left.0,5 \%\right)$. La aparición de un anillo rojo en la superficie indicó reacción positiva.

Identificación fenotípica con kits comerciales: la identificación fenotípica se llevó a cabo mediante el uso del sistema API 20 Strep (Biomerieux).

Otras determinaciones: se obtuvieron muestras de sangre con las que se realizaron extendidos, así como fragmentos de branquias, hígado, bazo, tubo digestivo y riñón. Pequeñas porciones de cada uno de estos órganos fueron fijadas en solución de Bouin y posteriormente deshidratadas e incluidas en parafina. Los cortes histológicos fueron coloreados con Hematoxilina-Eosina, PAS y Giemsa, para su análisis.

\section{RESULTADOS}

En el examen macroscópico se encontraron lesiones hemorrágicas en tegumento a nivel de cabeza,
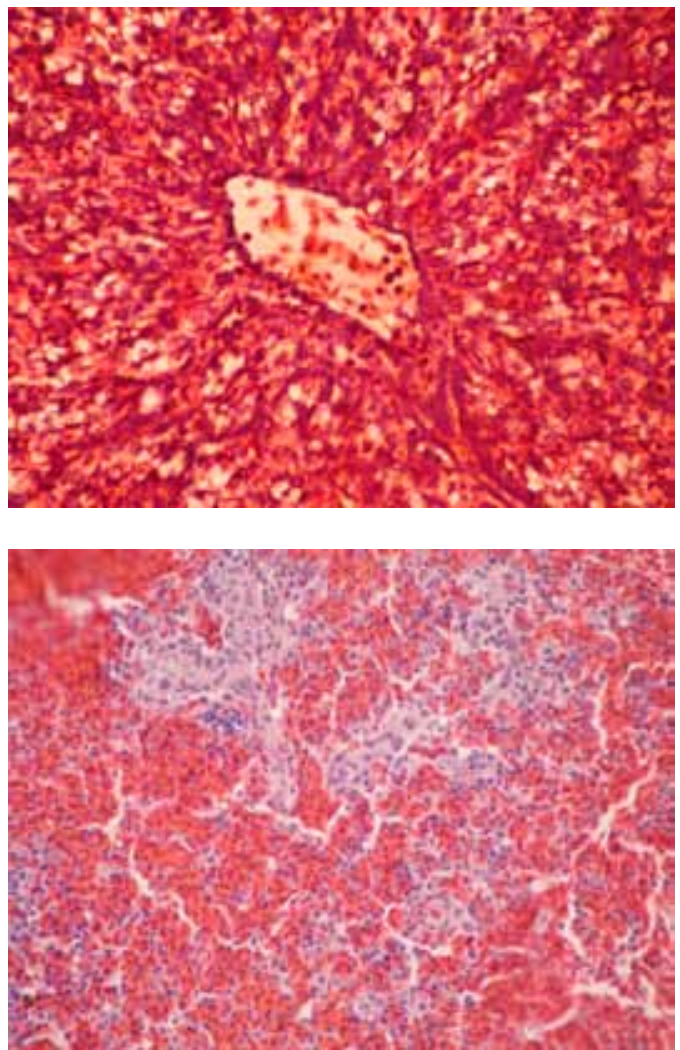

aletas y ojos, como también manchas puntiformes en superficie ventral, próximas al opérculo y almohadilla dentaria. Las lesiones internas observadas consistieron en la presencia de líquido ascítico y numerosas lesiones puntiformes en el hígado, el cual presentó disminución de su consistencia.

El análisis microscópico de las improntas del hígado determinó la presencia de cocos Gram positivos aislados, en diplos, cadenas cortas y grandes agrupaciones. Se observó el desarrollo de los microorganismos en el medio enriquecido a través del incremento en la turbidez respecto del medio sin inocular. La siembra por estría en placas conteniendo MRS agar permitió el desarrollo de colonias puntiformes y transparentes.

Las pruebas bioquímicas de los aislamientos obtenidos indicaron la presencia de bacterias esféricas Gram positivas aisladas, en diplos o pequeñas cadenas, catalasas negativas e incapaces de reducir nitratos y sintetizar triptófano a partir de indol, características
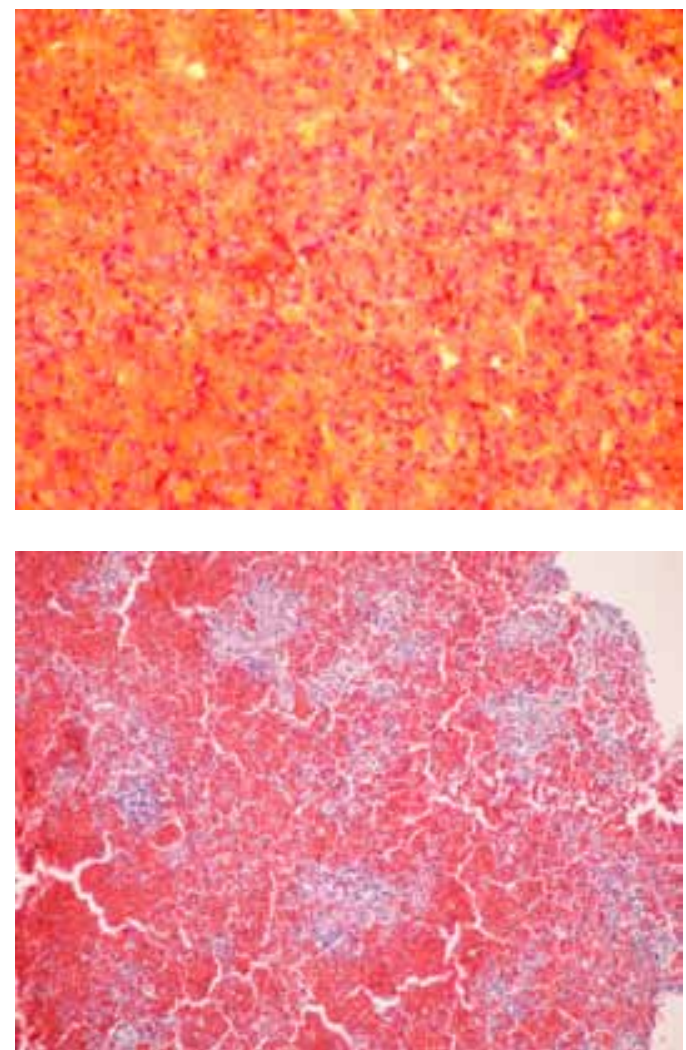

Figura 1. Hígado de P. reticulatum. Arquitectura parcialmente conservada, predominio de alteraciones vasculares, hemorragias, procesos degenerativos, áreas de necrosis y fenómenos regenerativos. Arriba: 40x, HyEGiemsa, Abajo izquierda: 40x, HyE, Abajo derecha: 20x, HyE. 

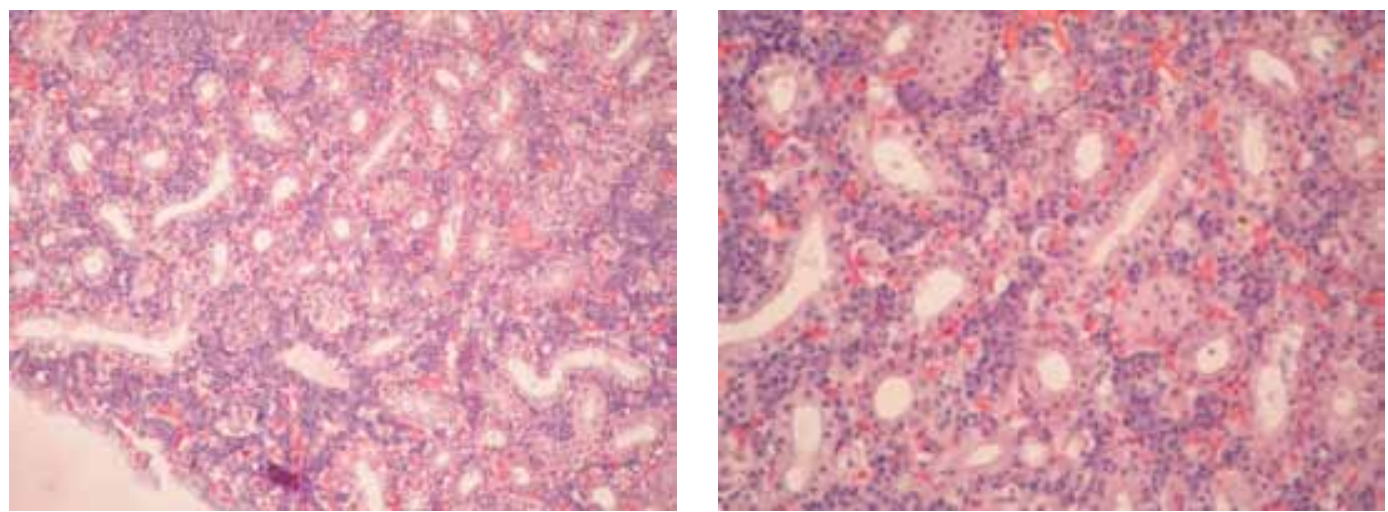

Figura 2. Riñón de P. reticulatum. Arquitectura conservada, fenómenos congestivos y hemorrágicos leves con invasión bacteriana y reacción inflamatoria escasa. Izquierda: 20x, derecha: 40x, HyE.
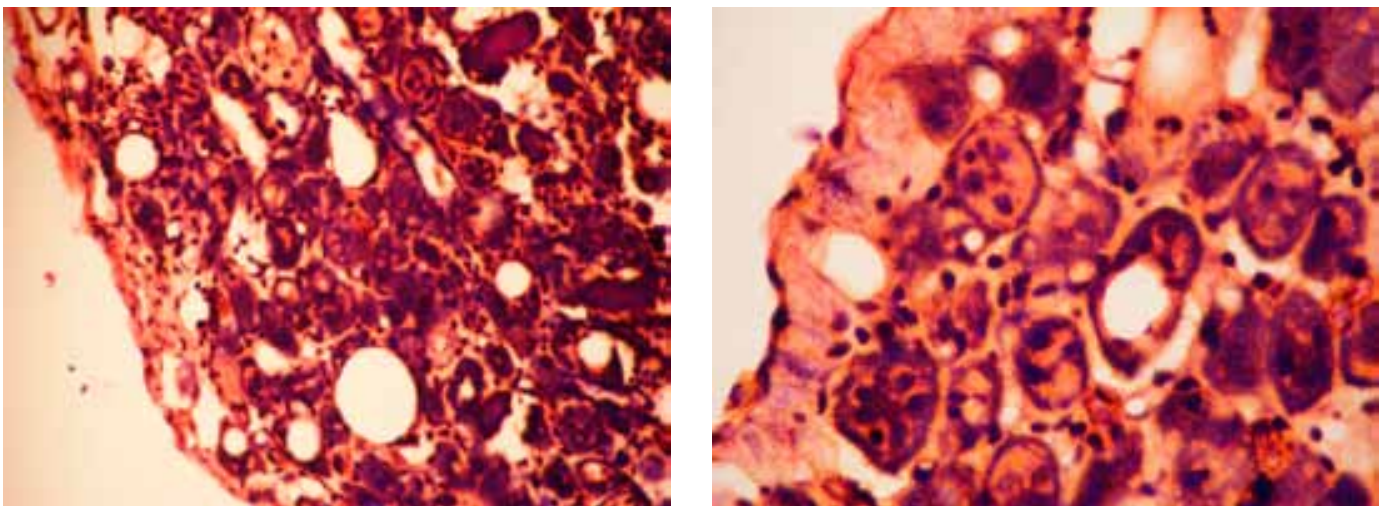

Figura 3. Bazo de P. reticulatum. Alteraciones vasculares, predominio congestivo y hemorrágico. Izquierda: 40x. Derecha: 100x. HyE-Giemsa.

propias de las bacterias ácido lácticas. Los resultados obtenidos con el sistema API 20 Strep se detallan en Tabla 1.

En el examen microscópico se observaron las branquias con arquitectura conservada y estructura laminar ordenada, detectándose congestión moderada. En el hígado la estructura estaba parcialmente conservada con predominio de alteraciones vasculares, fundamentalmente hemorrágicas de marcada intensidad y lesiones celulares intensas del parénquima. Se detectaron procesos degenerativos en diferentes etapas evolutivas, presencia de extensas áreas de necrosis, caracterizadas por pérdida de detalles celulares y núcleos picnóticos. Se registraron áreas de reacción tisular caracterizadas por fenómenos regenerativos. En el hígado se destacó invasión bacteriana con predominio de ubicación periférica. La reacción inflamatoria fue escasa, más evidente en la periferia del órgano y alrededor de vasos sanguíneos (Figura 1).

En el riñón la arquitectura se presentó conservada y se evidenciaron fenómenos congestivos y hemorrágicos leves, con invasión bacteriana y reacción inflamatoria escasa (Figura 2).

A nivel del tubo digestivo la arquitectura estuvo conservada. Tanto el epitelio como las estructuras glandulares se encontraron normales. En el bazo se observaron intensas alteraciones vasculares, predominio congestivo y hemorrágico (Figura 3). Los extendidos de sangre revelaron numerosos elementos figurados de la serie blanca con predominio linfocítico y plaquetario.

\section{DISCUSIÓN}

El notable desarrollo de la producción intensiva de peces produjo paralelamente un incremento progresivo en la incidencia y gravedad de las enfermedades infecciosas, especialmente las de etiología bacteriana ${ }^{32}$. A excepción de Renibacterium salmoninarum, las enfermedades producidas por bacterias Gram positivas recibieron siempre un menor grado de atención, por considerarse que el impacto productivo era poco relevante. Sin embargo, las enfermedades ocasionados por cocos y cocobacilos Gram positivos y catalasa negativos, aumentaron su frecuencia y virulencia.

La piscicultura en Argentina tuvo un notable desarrollo en la región nordeste, con numerosos productores de pacú, carpas, dorado, surubí, tilapia, sábalo, boga y salmón de río. Pseudoplatystoma reticulatum es una especie muy apreciadas para el consumo, por lo que el avances en el conocimiento de las patologías contribuye a evitar pérdidas por muerte o devaluación comercial de los peces enfermos, por el descenso de la producción o por los gastos de tratamiento, profilaxis y control sanitario que se deben realizar en las instalaciones afectadas.

En este trabajo se observa que el brote que ocasionó la mortandad de numerosos ejemplares se produjo al 
final de la primavera y afectó a ejemplares juveniles de Pseudoplatystoma reticulatum, lo cual estaría relacionado con lo descrito en algunos trabajos que reportan mayor susceptibilidad en ejemplares jóvenes ${ }^{32}$.

La lactococosis fue reportada en numerosas especies de peces en todo el mundo, ocasionando elevadas pérdidas económicas ${ }^{17,32}$, caracterizándose por producir una septicemia hemorrágica hiperaguda, que evoluciona cuando la temperatura del agua es superior a $15^{\circ} \mathrm{C}^{4,28}$.

El aislamiento permitió identificar bacterias esféricas inmóviles, Gram +, catalasa y oxidasa negativa. Usando API 20 se identificó a Lactococcus garvieae, cuyas propiedades bioquímicas fueron similares a las descriptas en otros trabajos ${ }^{2,29}$.

Las lesiones observadas en el surubi atigrado coinciden con las descriptas en brotes infecciosos por $\mathrm{Lac}$ tococcus. Las petequias y hemorragias en la superficie y órganos internos se producen por el daño en el endotelio vascular que ocasionan las toxinas ${ }^{32}$. Los principales órganos afectados son hígado, bazo, cerebro, vejiga natatoria, intestino, riñón y corazón ${ }^{24,26,27,28}$. Histológicamente se caracterizaron lesiones hemorrágicas, inflamatorias, necróticas y fibroplásicas en los distintos órganos afectados $2,4,5,6,10$.

Los microorganismos del género Lactococcus pasaron de considerarse patógenos oportunistas a patógenos emergentes ${ }^{1}$. Su importancia se extiende más allá de la acuicultura, ya que existe un creciente número de casos en los que se aísla e identifica esta bacteria en seres humanos. En el nordeste argentino es la primera vez que se reporta esta enfermedad.

\section{REFERENCIAS}

1. Aguado M. 2013. Caracterización y análisis funcional del genoma de Lactococcus garvieae. http://eprints.ucm. es/25117/1/T35311.pdf. Tesis Doctoral Universidad Complutense de Madrid.

2. Avci H, Aydogan A, Tanrikul TT, Birincioglu SS. 2010. Pathological and microbiological investigations in rainbow trout (Oncorhynchus mykiss Wlvaum, 1792) naturally infected with Lactococcus garvieae. Kafkas Univ Vet Fak Derg 16: S313-318.

3. Austin B, Austin DA. 1993. Bacterial fish pathogens: diseases in farmed and wild fish, $2^{\circ}$ ed., Ellis Horwood Limited, Chichester, Reino Unido.

4. Chang P, Lin C, Lee Y. 2002. Lactococcus garvieae infection of cultured rainbowtrout, Oncorhynchus mykiss in Taiwan and associated biophysical characteristics and histopathology. Bull Eur Ass Fish Paphol 22: 319-327.

5. Chen SC et al. 2002. Lactococcus garvieae, a cause of disease in grey mullet, Mugil cephalus L. in Taiwan. $J$ Fish Dis 25: 727-732.

6. Cheng W, Chen J. 1998. Isolation and characterization of an Enterococcus-like bacterium causing muscle necrosis and mortality in Macrobrachium rosenbergii in Taiwan. Dis Aquat Org 34: 93-101.
7. Collins MD, Farrow JA, Phillips BA, Kandler O. 1983. Streptococcus garvieae sp. Nov. and Streptococcus plantarum sp. Nov. J Gen Microbiol 129: 3427-3431.

8. Doménech A, Prieta J, Fernández JF, Collins MD, Jones D, Domínguez L. 1993. Phenotypic and phylogenetic evidence for a close relationship between Lactococcus garvieae and Enterococcus seriolicida. Microbiol 9: 63-68.

9. Doménech A et al. 1996. Streptococcosis in cultured turbot (Schophtalmus maximus) associated with Streptococcus parauberis. J Fish Dis 19: 33-38.

10. Eldar A, Ghittino C, Asanta L, Bozzetta E, Gloria M, Prearo M, Bercovier H. 1996. Enterococcus seriolicida is a junior synonym of Lactococcus garvieae, a causative agent of septicemia and meningoencephalitis in fish. Curr Microb 32: 85-88.

11. Eldar A, Ghittino C. 1999. Lactococcus garvieae and Streptococcus iniae infection in rainbow trout (Oncorhynchus mykiss): similar but different diseases. Dis Aquat Org 36: 227-231.

12. Eldar A, Gloria M, Ghittino C, Zlotkin A, Bercovier H. 1999. Biodiversity of Lactococcus garvieae strains isolated from fish in Europe, Asia, and Australia. Appl Environ Microbiol 65: 1005-1008.

13. Evans JJ, Klesius PH, Shoemaker CA. 2009. First isolation and characterization of Lactococcus garvieae from Brazilian Niletilapia, Oreochromis niloticus (L.), and pintado, Pseudoplathystoma corruscans (Spix \& Agassiz). J Fish Dis 32: 943-951.

14. Facklam R, Elliot J. 1995. Identification, classification, and clinical relevance of catalase-negative, gram-positive cocci, excluding the streptococci and enterococci. Clin Microbiol Rev 8: 479-495.

15. Fefer J, Ratzan K, Sharp S, Saiz E. 1998. Lactococcus garvieae endocarditis: report of a case and review of the literature. Diagn Microbiol Infect Dis 32: 127-130.

16. Ferrario C. 2013. Lactococcus garvieae and Morganella morganni: two bacterial models to study quality and safety of fish products. PhD Tesis, Università degli Studi di Milano, Italia, AGR/16, $101 \mathrm{p}$.

17. Ghittino C, Prearo M. 1992. Report of Streptococcosis in rainbow trout (Oncorhynchus mykiss) in Italy: preliminary note. Boll Soc It Patol Ittica 8: 4-11.

18. Harrigan WF, McCance ME. 1976. Laboratory methods in food and dairy microbiology, 1ra ed., Academic Press, Londres, $464 \mathrm{p}$.

19. Hirakawa T, Alves F, Cairo M, Rigon M, Abensur H, EImor M. 2011. Endocarditis por Lactcoccus garvieae: primer relato de caso de América Latina. Arq Bras Cardiol 97: 108-110.

20. Kusuda R, Kawai K, Salati F, Banner CR, Fryer JL 1991. Enterococcus seriolicida sp. nov., a fish pathogen. Int J Syst Bacteriol 41: 406-409.

21. Kusuda R, Salati F. 1999. Enterococcus seriolicida and Streptococcus iniae. In: Fish diseases and disorders (Woo PT, Bruno DW, Eds), 1ra ed., vol. 3, CAB Internat. Publ., Wallingford, Reino Unido, 944 p. 
22. Michel C, Nougayrede P, Eldar A, Sochon E, Kinkelin P. 1997. Vagococcus salmoninarum, a bacterium of pathological significance in rainbow trout Oncorhynchus mykiss farming. Dis Aquat Org 30: 199-208.

23. Morita $\mathbf{H}$ et al. 2011. Complete genome sequence and comparative analysis of the fish pathogen Lactococcus garvieae. PLoS ONE 6 (8): e23184, doi: 10.1371.

24. Múzquiz J, Royo F, Ortega C, Blas I, Ruiz I, Alonzo J. 1999. Pathogenicity of streptococcosis in rainbow trout (Oncorhynchus mykiss): dependence of age of diseased fish. Bull Eur Ass Fish Pathol 19: 114-119.

25. Nishiki I, Furukawa M, Matui S, Itami T, Nakai T, Yoshida T. 2011. Epidemiological study on Lactococcus garvieae isolates from fish in Japan. Fish Sci 77: 367-373.

26. Pereira F, Ravelo C, Toranzo AE, Romalde JL. 2004. Lactococcus gravieae, enemerging pathogen for the Portuguese trout culture. Bull Eur Ass Fish Pathol 24: 274279.

27. Prieta $\mathbf{J}$ et al. 1993. Lactococcosis de la trucha arco iris. Med Vet 10: 367-373.
28. Soltani M, Nikbakht G, Mousavi HA, Ahmadzadeh N. 2008. Epizootic outbreaks of lactococcosis caused by Lactococcus garvieae in farmed rainbow trout (Oncorhynchus mykiss) in Iran. Bull Eur Ass Fish Pathol 28: 201-212.

29. Tanrikul TT, Gultepe N. 2011 Mix infection in rainbow trout (Oncorhynchus mykiss Walbaum): Lactococcus garvieae and Vibrio anguillarum. J Anim Vet Adv 10: 10191023.

30. Teixeira LM et al. 1996. Phenotypic and genotypic characterization of a typical Lactococcus garvieae strains isolated from water buffalos with subclinical mastitis. Int $J$ Syst Bacteriol 46: 664-668.

31. Vela AI et al. 2000. Phenotypic and genetic characterization of Lactococcus garvieae isolated in Spain from Lactococcosis outbreaks and comparison with isolates of other countries and sources. J Clin Microbiol 38: 3791-3795.

32. Vendrell D, Balcázar JL, Ruiz I, Blas I, Gironés O, Múzquiz JL. 2006. Lactococcus garvieae in fish: a review. Comp Immunol Microb Infect Dis 29: 177-198.

\section{Asociación Cooperadora de la Facultad de Ciencias Veterinarias Universidad Nacional del Nordeste}

Personería Jurídica No 647/92 y 912/00

Sargento Cabral 2139

3400 Corrientes

Argentina

La Asociación Cooperadora de la Facultad de Ciencias Veterinarias de la UNNE fue constituida el 10 de diciembre del año 1991 como entidad de bien público, con el objeto de promover y coadyuvar las actividades científicas, educativas y culturales relacionadas con las Ciencias Veterinarias. En tal sentido, implementa acciones para colaborar con la enseñanza, extensión, actualización y difusión científica que realiza dicha casa de estudios.

\section{Beneficios que brinda a sus asociados:}

- Fotocopias con descuentos especiales en la Fotocopiadora Copias.com que funciona dentro del predio de la Facultad de Ciencias Veterinarias.

- Descuentos para la adquisición de libros de distintas editoriales.

- Descuentos especiales en otros rubros.

Tel. (0379) 4425753 interno 186•Página Web www.vet.unne.edu.ar•E-mail: cooperadora@vet.unne.edu.ar 